\title{
Review
}

\section{Fungi and fungal toxins as weapons}

\author{
R. Russell M. PATERSON* \\ Micoteca da Universidade do Minho, Centro de Engenharia Biológica, Campus de Gualtar, 4710-057 Braga, Portugal
}

\section{A R T I C L E I N F O}

\section{Article history:}

Received 19 December 2005

Received in revised form

27 February 2006

Accepted 1 March 2006

Corresponding Editor:

Nicholas P. Money

\section{Keywords:}

Biocontrol agents

Bioweapons

Mycotoxin decontamination

Mycotoxins

Mycotoxicosis treatments

Pharmaceuticals

Toxins

\begin{abstract}
A B S T R A C T
Recent aggressive attacks on innocent citizens have resulted in governments increasing security. However, there is a good case for prevention rather than reaction. Bioweapons, mycotoxins, fungal biocontrol agents (FBCA), and even pharmaceuticals contain, or are, toxins and need to be considered in the context of the new paradigm. Is it desirable to discuss such issues? None of the fungi are (a) as toxic as botulinum toxin from Clostridium botulinum, and (b) as dangerous as nuclear weapons. One toxin may be defined as a pharmaceutical and vice versa simply by a small change in concentration or a moiety. Mycotoxins are defined as naturally occurring toxic compounds obtained from fungi. They are the biggest chronic health risk when incorporated into the diet. The current list of fungal toxins as biochemical weapons is small, although awareness is growing of the threats they may pose. T-2 toxin is perhaps the biggest concern. A clear distinction is required between the biological (fungus) and chemical (toxin) aspects of the issue. There is an obvious requirement to be able to trace these fungi and compounds in the environment and to know when concentrations are abnormal. Many FBCA, produce toxins. This paper indicates how to treat mycotoxicosis and decontaminate mycotoxins. There is considerable confusion and inconsistency surrounding this topic which requires assessment in an impartial and scientific manner.
\end{abstract}

(c) 2006 The British Mycological Society. Published by Elsevier Ltd. All rights reserved.

\section{Introduction}

Recent mass attacks on citizens make it necessary to consider the implications of fungi as weapons. Because of the increased public interest and media attention regarding bioweapons, there is increased public pressure on relevant authorities to assess whether occurrences of food contamination are malicious acts (Elad 2005). The economic consequences of a potential attack can be huge, as evidenced after the recent attacks in the US (Lenain et al. 2002). It is more provident to prevent a bioweapon attack from happening in the first place, than to prepare a response. There is the dilemma of whether to declassify essential information with a view to preventing aggressive acts, or to disclose essential data to increase public awareness but inadvertently make it available to the aggressor. A compromise has to be drawn. However, considering the amount of public information already available, the rubicon may have been crossed. Somewhat subjectively drawn biosecurity guidelines have been prepared, which are essential reading to anyone working with fungi that could be considered as possible threats (Tucker 2003). They have highly significant implications that could severely limit research in the field, including determining which individuals do the work, irrespective of ability or qualifications. One wonders how representative the views of a publication are when the disclaimer states,

\footnotetext{
* Corresponding author.

E-mail address: russell.paterson@deb.uminho.pt 0953-7562/\$ - see front matter @ 2006 The British Mycological Society. Published by Elsevier Ltd. All rights reserved. doi:10.1016/j.mycres.2006.04.004
} 
"The views expressed in this report are those of the author alone. They do not necessarily reflect views of the United States Institute of Peace". A public debate is required on this area. Some well-known reports have claimed recently that aflatoxins were placed in warheads for use by Iraqis, although the effect of such a limited amount of aflatoxins dispersed in this manner would be minimal.

Furthermore, the use of fungi in technologies (e.g. biocontrol) requires revision because of the heightened security awareness. This also has ramifications for the health and safety of those who use these organisms for mass production or in non-sterile conditions. There is a temptation to ignore the issue, but this is inappropriate when information is becoming increasingly available (Bennet \& Klich 2003; Miller et al. 2005; Paterson \& Lima 2005; Stark 2005), in addition to the dubious mixture of the informative (Locasto et al. 2004) and illegitimate material on the World Wide Web. A great deal is know about botulinum from Clostridium botulinum, which is the most toxic compound in the world (human lethal dose $0.2-2.0 \mu \mathrm{g} \mathrm{kg}^{-1}$ ). Fungal toxins although less toxic can be used as weapons. Some other potential bioweapons are the smallpox virus, Variola major (Shannon 2004), which resulted in a $30 \%$ fatality rate in previous epidemics, but can be higher in vulnerable groups; and Mycobacterium leprae which causes leprosy and is rarely lethal.

How do fungi compare? Simply to obtain some level of calibration at the extremes, they are not as dangerous as nuclear weapons. Ease of conversion to a weapon is a crucial factor (i.e. "weaponization"). The number of medically important fungi is lower than, for example, bacteria. Obvious growth of fungi on animals is called mycosis and they are primary pathogens (e.g. Histoplasma capsulatum) (Bennet \& Klich 2003). Opportunistic pathogenicity is associated with immunocompromised people. Interestingly, Aspergillus fumigatus is both: causing Farmers' lung disease, where one assumes the "farmers" were otherwise healthy (presumably the fungal loads in these cases were enormous), and causing $90 \%$ of fungal infections in immunocompromised patients (Sheppard et al. 2004). Mycosis can range in severity from athlete's foot to aspergillosis. Finally, dietary, respiratory, dermal and other exposure to mycotoxins is called mycotoxicosis.

There is an apparent similarity between fungal biocontrol agents (FBCA) and weapons, in that toxin-producing fungi are mass-produced and, for example, sprayed onto crops. Which raises the question, what are the natural levels of fungi and toxins in the environment (Gonçalves et al. 2006)? Interestingly, pharmaceuticals from fungi are also relevant to the discussion. The difference between a compound being a toxin or a drug may be a shift in a decimal point of concentration or a change in a simple moiety. How these compounds are classified depends to some extent on the prevailing "climate". For example, mycophenolic acid, ergot alkaloids, penicillin, and perhaps patulin, can be either toxins or drugs.

There has always been great interest in toxins from the macrofungi (e.g. mushrooms): Scientific endeavour started in mycotoxins with the discovery of aflatoxins in the 1960s. However, it is an extremely complex field largely due to its multidisciplinary nature. "Experts" commonly pontificate on areas in which they are not particularly qualified.

\section{Mycotoxins (Fig 1)}

There is little to better the US Council for Agriculture Science \& Technology (CAST) (2003) report on this topic and it is essential reading. However, Bennet and Klich (2003) are adroit. Paterson et al. (2004) may be worth consulting for some additional insights (e.g. the taxonomy of the fungi). An awareness of Hazard Analysis and Critical Control Point (HACCP) approaches for control is important (Aldred \& Magan 2004; Paterson 2006a). However, HACCP protocols were developed<smiles>CC1Cc2c(Cl)cc(C(=O)NC(Cc3ccccc3)C(=O)O)c(O)c2C(=O)O1</smiles>

Ochratoxin A

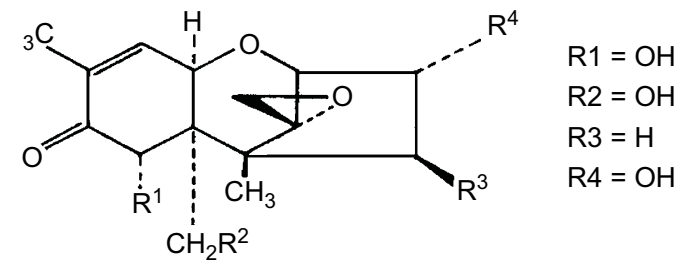

DON
Patulin<smiles>O=C1C=C2C(=CCOC2O)O1</smiles><smiles></smiles>

Aflatoxin $B_{1}$

Fig 1 - Chemical structure of some myocotoxins. 
to prevent unintentional contamination; further control and analytical steps may be required for intentional contamination. The universality of applying HACCP has been questioned (Sperber 2005).

Mycotoxins are (a) below microbiological, some phytotoxins and phycotoxins, and (b) above anthropogenic contaminants, pesticide residues, and food additives in terms of acute health risks. Significantly, they are the highest chronic risk factor in the diet (Kuiper-Goodman 2004). However, chronic effects are of little interest to weapon manufacturers, although they are of course relevant in cases of longer-term exposure. Presumably, the phytotoxins referred to are toxins from plants and not toxins from microbes that affect plants. It is not totally clear whether toxins (e.g. amanitin) from macroscopic fungi would be considered as mycotoxins or something else (e.g. erroneously as phytotoxins). Furthermore, some fungal metabolites are probably more toxic than mycotoxins (e.g. aflatoxins), which are simply not detected in the environment (see Cole \& Schweikert 2003a,b; Cole et al. 2003). These may be revealed through natural product screenings for drugs where toxic compounds are removed from further screens at early stages in the process.

Fig 2 presents a Venn diagram of the overlapping relationship between mycotoxins, pharmaceuticals, FBCA toxins, and fungal biochemical weapons, which illustrates clearly that the same compound can be represented in different fields. Presently, there are few metabolites considered to be mycotoxins out of potentially thousands. There are probably more toxic metabolites from FBCA than there are mycotoxins in this narrow definition but only a few (i.e. three) of them (Table 1) can be considered as mycotoxins. A minute percentage of toxic fungal metabolites have been considered seriously as weapons: two (aflatoxins and T-2 toxin; Fig 3) are obviously mycotoxins, whereas one is a toxin from a macroscopic

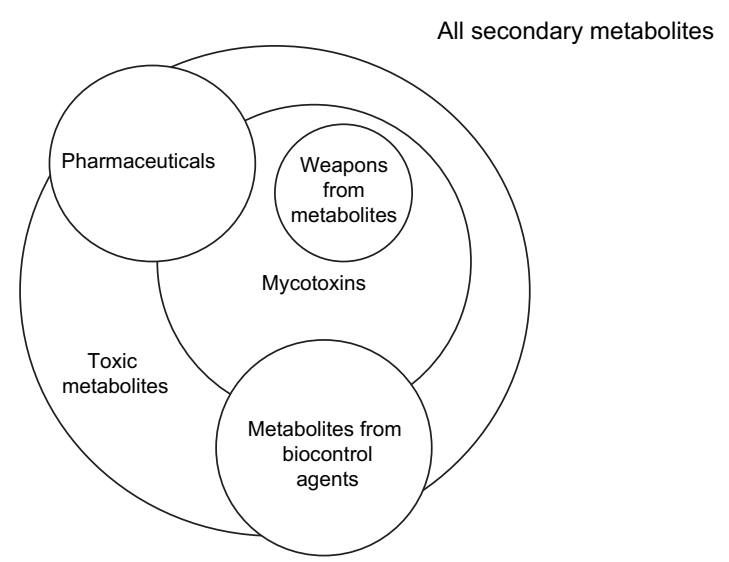

Fig 2 - Venn diagram of the relationship between fungal metabolites in terms of toxicity. The sizes of the circles are in proportion to the actual number of compounds only in a general manner.

fungus, i.e. amanitin, as mentioned previously. This differs from mycotoxins as the fungus is intentionally eaten, although it has been used to poison food and again the distinctions become blurred. Paterson \& Lima (2005) list the ergot alkaloids (CAST 2003) as other possibilities.

Mycotoxins are a somewhat exclusive group of low molecular weight compounds that are present in foods, and affect animals (e.g. humans). They are produced by filamentous fungi, but the fungi may no longer be present in the food. It is important to realise that mycotoxins are not the most effective weapons. For example, other toxins from fungi, which are not found in food, may be more toxic. For this reason a sound knowledge of which fungi produce which toxins (i.e. fungal chemotaxonomy) is crucial.

Table 1 - Approximate classification of some toxins of fungi

Toxin Grouping

\begin{tabular}{|c|c|c|c|c|}
\hline Mycotoxin & Pharmaceutical & Weapon & $\begin{array}{l}\text { Fungal biocontrol } \\
\text { agent toxin }\end{array}$ & $\begin{array}{c}\text { Other } \\
\text { entomopathogen toxin }\end{array}$ \\
\hline
\end{tabular}

\begin{tabular}{|c|c|c|}
\hline Aflatoxins & $t^{\mathrm{a}}$ & \\
\hline Ochratoxin A & $+^{a}$ & \\
\hline Cytochalasins & + & \\
\hline Beauvericin & + & \\
\hline Eniantins & + & \\
\hline \multicolumn{3}{|l|}{ Destruxins } \\
\hline \multicolumn{3}{|l|}{ Oosporein } \\
\hline Moniliformin & + & \\
\hline \multicolumn{3}{|l|}{ Efrapeptins } \\
\hline \multicolumn{3}{|l|}{ Beauveriolides } \\
\hline Amminita toxins & $++^{\mathrm{b}}$ & \\
\hline Patulin & $+^{a}$ & $+?^{c}$ \\
\hline Mycophenolic acid & + & + \\
\hline Penicillin & + & + \\
\hline $\mathrm{T}-2$ toxin & $+^{a}$ & \\
\hline Ergot alkaloids & $+^{a}$ & + \\
\hline
\end{tabular}




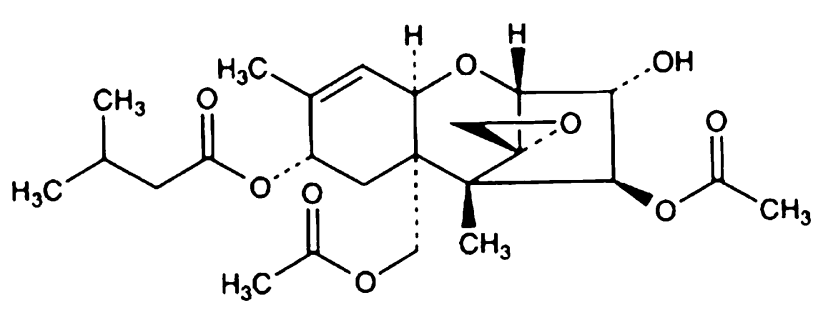

Fig 3 - Chemical structure of T-2 toxin.

Difficulty exists in defining mycotoxins in a few words. Some 300-400 are now recognised, although only about a dozen groups receive regular attention as threats to human and animal health. The incidence of mycotoxicosis may be more common than suspected. It is "easy" to attribute the symptoms of acute mycotoxin poisoning to other causes, but it is difficult to prove that cancer and other chronic conditions are caused by mycotoxin exposure. The scientific quality of the mycotoxin literature is variable. There has been much repetition and an imprecise use of jargon, especially in toxicology (Bennet \& Klich 2003). This is in part due to the multidisciplinary nature of mycotoxin research, which involves analytical chemistry, toxicology, taxonomic mycology, hygienic measures, microbial physiology, epidemiology, and increasingly weaponry. Too often experts in one field have felt the need to improvise in another.

\section{Bio/chemical weapons}

Fungal toxins as weapons or "expressions of discontent" are taken seriously. They can be used intrinsically as weapons, which could be employed by governments and/or small groupings of individuals. However, an individual could use them in a revenge attack on an employer for example. The supposed governmental deployments have ranged from the suspected use of T-2 toxin by the former Soviet Union (the evidence for this is very thin) to the development of aflatoxins by Iraq (the efficacy of which is minimal) (Bennet \& Klich 2003). (N.B. T-2 toxin appears to be a valid weapon.) It is axiomatic to state that any government would be interested in developing such weapons if they had the desired characteristics.

The factors that are fundamental to produce a serviceable chemical or biological weapon are: (1) efficient manufacture; (2) ease of conversion to a weapon ("weaponization"); (3) longevity of the organism or toxin in storage; (4) efficient dispersal; and (5) stability when exposed to the environment. Other factors are concealment and ability to obtain the toxin or organism.

In the early twentieth century, biotoxins were investigated militarily, and were rejected because of the difficulty in conversion to weapons. However, there has been renewed interest with developments in biotechnology. It has been stated that the US has no current offensive biological weapons capability. They are less expensive than nuclear and conventional chemical weapons and may appeal to countries or terrorist organisations where cost is an important issue (Locasto et al. 2004).
Of course, there is a dilemma surrounding a discussion of fungal toxins in relation to weapons. Will the information encourage their use and development, or do the reverse and provide appropriate information on how to deal with the potential threat? In any case, there are many reports in the public arena, including those of a dubious nature on the World Wide Web. Effectively the line has been crossed and a large amount of literature is available.

\section{Biological per se}

Fungi as biological agents of war are not tenable currently except perhaps for Coccioides immitis (Shannon 2004). Those that cause human disease are too slow-acting and would only affect selected members of the population or have to be applied in vast numbers if they were to be effective at all. An allergic response is hardly the stuff of weapons. Many fungi are not pathogens, but produce toxins (Cole \& Schweikert 2003a,b; Cole et al. 2003). Conceivably, Aspergillus flavus could be sprayed onto crops, but this is a ridiculous concept, as what would be the results? A single destroyed crop at most (however, see genetically engineered fungi section). To have a widespread effect the resources required would have to be enormous, even at the level of supplying enough growth medium for the fungus (Stark 2005). However, the use of Fusarium oxysporum to kill coca plants and hence cocaine manufacturing, is an interesting comparison (Connick et al. 1998; de Vries 2000). A list of the toxins reported from this species is provided in Table 2.

\section{Biochemical per se (toxins)}

Biotoxins, like chemical weapons such as mustard gas, need to be made from precursors (ethylene and $\mathrm{Cl}_{2}$ in the case of mustard gas) under the appropriate conditions. For example, a growth medium containing carbon and nitrogen as the predominant precursors must be inoculated with a live fungus in a suitable vessel (e.g. a bioreactor). The toxin is produced as a consequence of the metabolism of the fungus and is then purified. This can be undertaken in large bioreactors thus producing a large amount of toxin. So the toxin is a chemical and has no living component.

Biotoxins have inherent limitations to their manufacture in sufficient quantities as weapons. It is a small-group assassination tool as only a little can be dispersed effectively in enclosed areas. Equally, a revenge attack by a disgruntled employee is a possibility such as on a water distribution system (Mays 2004). It may be worthwhile illustrating the problem with an example.

The first report of the (presumably) natural-occurrence of a mycotoxin, or indeed any fungal secondary metabolite, in water was by Paterson et al. (1997), which was written after attempting to produce aflatoxin in water using a bioreactor. It was discovered twice that the control water, which was from a water tank used to serve the laboratory where the work was undertaken, contained aflatoxins $\left(B_{2}, G_{2}\right)$. The water, which had been inoculated with Aspergillus flavus and incubated, did not (Paterson unpubl.). Presumably, the fungus had degraded the aflatoxins present and hence they were no longer detectable. This raises the question, how did the 


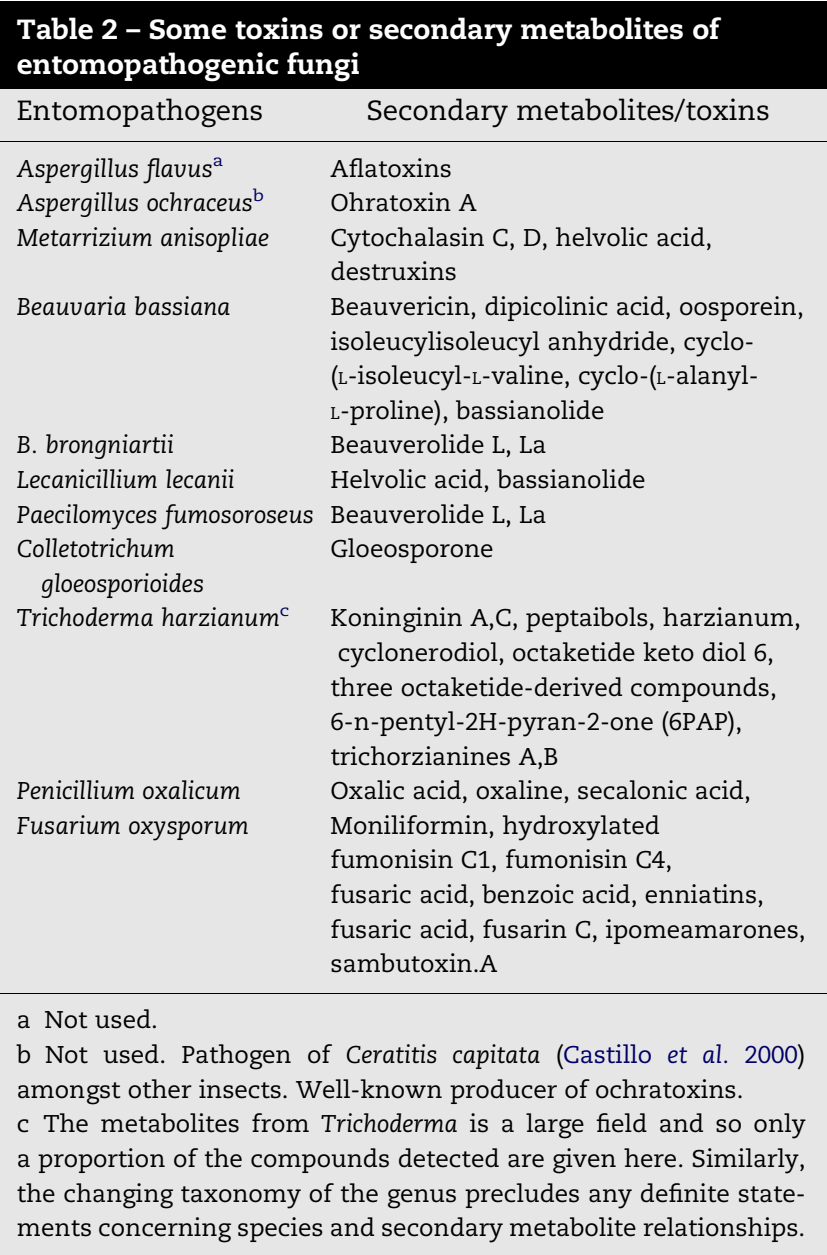

aflatoxins arise in the control water? An A. flauus strain was isolated from the control water thus indicating how the aflatoxins may have arisen. A. flauus is not associated with producing aflatoxin Gs and so another contaminant may have been present. However, it is not known whether this was from the natural growth of a fungus or from the intentional addition of fungus or toxins from a disgruntled employee. Certainly, numerous fungi were available at this particular organisation. The water was used for a variety of purposes, such as washing benches, cleaning floors, and supplying showers, so the levels of aflatoxins would have accumulated over the years and would have been breathed in. Indeed, it could have been drunk. Only a survey of similar water tanks would indicate if this contamination was normal or deliberate.

In addition, T-2 toxin can be used as a food or water-borne (Paterson \& Lima 2005) poison. Currently, T-2 toxin is the only biologically active toxin effective through dermal exposure, respiratory, and gastrointestinal (GI) portals. Tissues involved in high cellular turnover (e.g. GI and respiratory epithelium, bone marrow cellular elements) are the most susceptible to the toxin (Locasto et al. 2004).

The threat of toxins and particularly mycotoxins are made apparent in Garber et al. (2005), Miller et al. (2005), and Stark (2005). The current terrorist tactics have shifted attention to protection of food supplies, and consequently, it is a worldwide concern for the 21 st century. The impact on society could be anything from low to "catastrophic". A huge range of actions and programmes are being developed and implemented to prevent, deter, and respond to potential attacks. For example: (1) enhanced laboratory capability; (2) advanced tracking; (3) increased examinations; (4) enhanced surveillance; (5) more training; (6) recovery plans; and (7) new medical treatments. The above authors mention that mycological and chemical sampling and detection methodologies need to be geared up. Inactivation of mycotoxins and decontamination of food plants require urgent consideration. Foods need to be ranked in terms of vulnerability to attack, as do the risks to people when foods are intentionally contaminated. The development of algorithms to differentiate natural from unnatural food contamination (Paterson \& Lima 2005) are required. International and national collaborations are essential. Finally, biomarkers in humans and animals need to be further developed with particular relevance to metabolomics (Paterson 2006b).

$\alpha$-Amanitin from Amanita phalloides, amongst others, is a big concern as it is extremely toxic, water soluble, and heat stabile (Garber et al. 2005). However, mass production would presumably be limited to solid substrate bioreactors if basidiomes were used or conventional bioreactors if mycelium can produce the compound. The background counts of amanitin and T-2 toxin were useful as a demonstration of what is required to be done to distinguish abnormal from normal concentrations of biotoxins. However, in some cases the background was higher than the spiked samples. In some ways the paper by Stark (2005) is both alarming and reassuring in what is possibly an overly dramatic paper in the introduction at least. The use of mycotoxins as large-scale tactical weapons is impractical but could be used in the sabotage arena. It is also reassuring that antidotes do already exist for some mycotoxins.

The concept of liver cancer from aflatoxin as a battlefield weapon is ludicrous, and the reports of acute toxicity are uncertain. The compound as a weapon can hardly be taken seriously apart from a suggested psychological effect, although acute poisonings have been considered. Conversely, the exposure to T-2 toxin of a few milligrams is potentially lethal. The concept of mass production of the compound may be impractical simply because of the quantity of growth medium required (Stark 2005) and it would take a vast amount of malt extract to produce significant amounts. Although whether another cheaper and more plentiful medium could be used is a possibility, although what this might be is uncertain.

\section{Water}

Drinking, or non-drinking water may be an effective medium for mycotoxin dispersal as a weapon and is worthy of a separate section. The threat from contaminated drinking water is obvious. In the case of non-drinking water, the toxin could be spread by spray from a shower and then breathed in. Work places where a lot of water may be employed, such as farms, or a car wash, could be susceptible. The levels of safety required for water for livestock may be considerably lower than water for human consumption, so this could be a potential route of attack. In an example, stored water that was demonstrated to contain aflatoxins was used to clean laboratories 
and so contaminated dust could be spread accumulatively. Toxins in water are possible naturally, unnaturally, and from inoculation of fungi (Paterson \& Lima 2005).

\section{FBCA}

These are given more space than might be merited in a general review of bioweapons. However, there are some interesting parallels between the concepts of fungal weapons us FBCA. This technology needs to be reassessed. FBCA are of great interest to mycologists and issues are now being raised regarding safe use (Skrobek et al. 2005) which are especially relevant in the current high-security climate. For example, the procedures involve applying natural fungal pathogens to crops in the field or in storage, to control insect pests or disease. They have been advocated by some as an effective and more particularly, an environmentally sound means of controlling pests, disease, and undesirable organisms. A major concern regarding these preparations is how safe they are in terms of toxin production (Skrobek et al. 2005; Strasser et al. 2000). After all, they could affect the general population and workers producing the FBCA. They have been introduced or tested disproportionately in developing countries where, ironically, the mycotoxin problem is worse.

In a large study on risk assessment Strasser et al. (2000) did not consider the situation of compounds produced by BCAs, which are already-known mycotoxins. For example, cytochalasins are not discussed, although they are produced by Metarhizium anisopliae, which is considered by the authors. Cytochalasin D (Fig 4), in particular, is classified as very toxic. If it is satisfactory to use the fungi mentioned because the levels in the environment would be low, is it equally safe, for example, to use Aspergillus flavus which produces aflatoxins or A. ochraceus which produce ochratoxin A (OTA)? There needs to be some consistent scientific thinking here.

An example of a FBCA is the use of Fusarium oxysporum (as F. oxysporum f. sp. exythoroxilum) to kill coca plants in certain Latin American countries (Connick et al. 1998; de Vries 2000). Interestingly, the idea is to introduce a disease rather than a cure. The ultimate objective is to stop the manufacture of cocaine. Table 2 lists the toxins associated with the fungus, and it appears that the effect of this FBCA has not had sufficient consideration. The concepts of what constitutes this taxon are complex, and toxin production from different special forms has not been clarified satisfactorily. The technology is similar to what would be required for the production of a bioweapon.

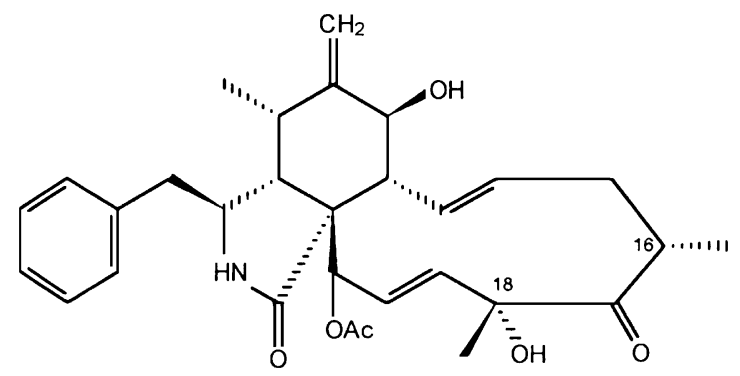

Fig 4 - Chemical structure of cytochalasin D.
Mycotoxins have been ranked as the most important chronic risk factor in the diet above pesticide residues, synthetic contaminants, plant toxins, and food additives as mentioned previously (Bennet \& Klich 2003). They are considered more acutely toxic than pesticides. Those interested in applying this technology need to collaborate more fully with those who know how to control the secondary metabolism that produces the toxins, although control of production in the field will be difficult in a predictable manner.

\section{Pharmaceuticals}

The surprising fact that penicillin can be detected in foodstuffs has been highlighted recently (Laich et al. 2002). Obviously, when there is such concern about resistant bacteria, and sensitised patients, this needs serious consideration. The penicillins are also inherently toxic as many who have been on a course of the drug will testify. Again the issue is concentration; exposure to low concentrations causes some of the resistance problems. Another example is mycophenolic acid. This has only recently has been adopted as an immuno-suppressive pharmaceutical but has been longer known as a minor mycotoxin. Patulin may also be worth mentioning as it was originally described as a pharmaceutical but was considered to be too toxic for general use. However, it is now firmly in the mycotoxin camp.

\section{Future trends}

I predict that there will be more compounds considered as mycotoxins within $10 \mathrm{y}$; the trend is for more compounds to be included. Mycotoxins will become acceptable only at ever decreasing concentrations tending towards background levels. Increasing numbers will be shown to be toxic and present in different foods. So the mycotoxin circle in Fig 2 will become wider. I also predict that the number of FBCAs will decrease. Also, compounds from these may begin to be considered more seriously as mycotoxins (e.g. destruxins), and consequently, will result in fewer FBCAs. The trend for weapons is difficult to predict. It may be that they will begin to be considered as "not effective". Alternatively, they could expand into the "non-mycotoxin toxins" (e.g. Cole \& Schweikert 2003a,b; Cole et al. 2003). This type of activity is reported in the literature but the compounds are not usually found, or investigated, in food.

\section{Genetically modified fungi (GMO)}

It is possible to speculate about altered strains that could be more virulent or produce higher yields of toxins than the wild-type. Countries with large resources could perhaps develop such strains. It is something of a worst case scenario. However, it is probably only a matter of time before such an organism exists considering the developments in genetics, if it does not already. In developed countries transfer of cultures from genetic resource collections to second parties involves the completion of a "material transfer agreement" which forbids genetic modification of the received cultures unless containment is increased to accommodate the GMO. However, it 
is not clear what GMOs mean. Does it specifically require that genetic information is transferred from one taxon to another? A useful example is how the titre of penicillin has been vastly increased from the original meagre amounts by strain improvement techniques and this could perhaps be undertaken for toxin production.

\section{Security of laboratories and obtaining pure mycotoxins}

This issue is discussed in depth in Tucker (2003), although some controversial issues are raised, such as which individuals and laboratories could be allowed to work in the field, and can the degree of control possible in the USA be applied to other countries. The degree of risk pertaining to each situation needs to be determined. Put crudely, does working with certain pathogenic bacteria equate in safety terms with working with fungi? Is the risk from the fungus (i.e. a biological one) or the toxin produced (i.e. a chemical one)? If it is the toxin then would not chemical security procedures be more appropriate and which have been established longer? There are currently discussions on biosecurity within biological resource centres, which, in the case of fungi, roughly translates to "culture collections". Some relevant concerns are raised by the World Health Organisation (WHO 2003). Global events recently have underlined the need to protect laboratories and the materials they contain in a way that will protect people, livestock, the environment, and agriculture. However, there are distinctions between laboratory biosecurity and biosafety. Biosecurity measures prevent intentional release, loss, misuse, theft, and diversion of pathogens and toxins. In contrast, biosafety is containment procedures that are implemented to prevent unintentional exposure of pathogens/toxins or accidental release. Security precautions need to become routine laboratory practice, according to the WHO.

In addition, anyone trying to obtain pure mycotoxins from the chemical companies will realise that it has become more difficult. Often legitimate proof of use is required. Presumably this is because of security and not from an increased level of concern for workers health per se. There is now also more paper work and security surrounding sending toxigenic cultures between laboratories. Concern with respect to health and safety is mostly related to the mass production of fungi, especially dried conidia, which can be breathed in easily. Similarly a great deal of care is required when handling purified and dried toxin preparations.

\section{Mycotoxicosis treatment}

Supportive therapy for mycotoxicosis is improved diet and hydration of patients (Locasto et al. 2004). Taking super-activated charcoal orally may be effective if toxins are swallowed (e.g. T-2 toxin). The route of entry and dose indicate the clinical course for T-2. From a detailed study of OTA toxicity and activation metabolism of aflatoxin B1, it was discovered that the sweetener aspartame is very protective against OTA intoxication, and that Oltipraz effectively protects against $\mathrm{AFB}_{1}$ acute toxicity and carcinogenicity (Stark 2005). Oltipraz has been tested in China on populations exposed to aflatoxins (Bennet
\& Klich 2003). Some strains of Lactobacillus effectively bind dietary mycotoxins and may also be an effective treatment.

\section{Mycotoxin decontamination}

Biotoxins from fungi would be difficult to remove from food and water (Paterson \& Lima 2005). The methods devised by Castegnaro et al. (1991) would at least be effective for the mycotoxin contamination of environments such as rooms. The most usual procedure for decontamination is washing with bleach, which effectively oxidises most aflatoxin and some other mycotoxins (Stark 2005). Potassium permanganate under alkaline conditions appears to be effective for a wider range of mycotoxins and for more situations than bleach, a point that appears to have been overlooked. The use of an enzyme to degrade the toxin might be practical technically but is probably not yet feasible as a routine or emergency procedure. Sharpira (2004) provides extensive details on decontamination of foods.

\section{Some priorities}

It needs to be recognised that it is the low molecular weight toxins from fungi, not the fungus, that present the biggest threat, apart from the remote possibility of a genetically engineered fungus causing unconstrained damage. There is a requirement to have an understanding of what are normal levels of fungi and toxins in the environment (Gonçlaves, et al. 2006; Paterson \& Lima 2005; Paterson in press). Which toxins are acute in nature (e.g. T-2 toxin) and which are more likely to be chronic (e.g. aflatoxins) should be established. Methods for analysing the toxins are required. Fortunately, some excellent methods for multimycotoxin analysis, based on chromatography, exist as are described in Paterson \& Lima (2005). Some basic protocols are provided in that publication for water. Single method procedures for hundreds of compounds are of particular value and standardised protocols could be based on these. These can be compared with PCR methods as detailed in Paterson (2006b). There are vast amounts of data on the levels of the more well-known mycotoxins in a variety of foods. CAST (2003) is a good starting point, but there are more data from various surveys. It is worthwhile listing those compounds that are water soluble as this will be a crucial factor in water contamination and also some foods. Information as to which foods are usually contaminated with particular mycotoxins and which would not normally be expected is essential. Furthermore, it is crucial to appreciate the uncertainties in this form of analysis (CAST 2003; Whitaker \& Johansson 2005). For example, samples of corn contaminated with aflatoxin at $10 \mathrm{ngg}^{-1}$ and $10000 \mathrm{ng} \mathrm{g}^{-1}$ are estimated to vary in a repeated subsequent analysis by 0-33.9 $\mathrm{ng} \mathrm{g}^{-1}$ and 8992-11008 $\mathrm{ng} \mathrm{g}^{-1}$, respectively. So one can immediately understand the problem of deciding if a sample was intentionally contaminated.

\section{Conclusions}

Low molecular weight toxins from fungi need to be recognised as the biggest threat as bioweapons. Fungi are perhaps not 
a significant threat, although some toxins from them are. However, various factors needs to be considered and not simply overall toxicity or notoriety. Ease of "weaponisation" is important. However, T-2 toxin is a significant threat. Toxins, other than the well-known mycotoxins, require consideration. It is fundamental to be able to differentiate between normal and abnormal concentrations of toxins or fungi in the food and water supplies.

\section{Acknowledgements}

R.R.M.P. is funded by grant SFRH/BPD/14923/2004 from the Fundação para a Ciência e a Tecnologia, Portugal.

\section{R E F E R E N C E S}

Aldred D, Magan N, 2004. The use of HACCP in the control of mycotoxins: the case of cereals. In: Magan N, Olsen M (eds), Mycotoxins in Food Detection and Control. Woodhead Publishing, Cambridge, UK, pp. 139-171.

Bennet JW, Klich M, 2003. Mycotoxins. Clinical Microbiological Reviews 16: 497-516.

CAST, 2003. Mycotoxins: Risks in Plant, Animal, and Human Systems. Council for Agricultural Science and Technology, Ames, IA. http://www.cast-science.org/cast/pub/Mycotoxins.pdf.

Castillo M-A, Moya P, Hernández E, Primo-Yúfera E, 2000. Susceptibility of Ceratitis capitata Wiedemann (Diptera: Tephritidae) to entomopathogenic fungi and their extracts. Biological Control 19: 274-282.

Castegnaro M, Barek J, Fremy JM, Lafontaine M, Miraglia M, Sansone EB, Telling GM, 1991. Laboratory decontamination and destruction of carcinogens in laboratory wastes. Some mycotoxins. IARC Scientific Publications No. 113. International Agency for Research on Cancer, Lyon.

Connick Jr WJ, Daigle DJ, Pepperman AB, Hebber KP, Lumsden RD, Anderson TW, Sands DC, 1998. Preparation of stable, granular formulation containing Fusarium oxysporum pathogenic to narcotic plants. Biological Control 13: 79-84.

Cole RJ, Schweikert MA, 2003a. Handbook of Fungal Secondary Metabolites. Volume 1. Academic Press, San Diego.

Cole RJ, Schweikert MA, 2003b. Handbook of Fungal Secondary Metabolites. Volume 2. Academic Press, San Diego.

Cole RJ, Jarvis BB, Schweikert MA, 2003. Handbook of Fungal Secondary Metabolites. Volume 3. Academic Press, San Diego.

Elad D, 2005. Risk assessment of malicious biocontamination of food. Journal of Food Protection 68: 1302-1305.

Garber EAE, Eppley RM, Stack ME, McLaughlin MA, Park DL, 2005. Feasibility of immunodiagnostic devices for the detection of ricin, amanitin, and T-2 toxin in food. Journal of Food Protection 68: 1294-1301.

Gonçalves AB, Paterson RRM, Lima N, 2006. Survey and significance of filamentous fungi from tap water. International Journal of Hygiene and Environmental Health 209: 257-264.

Kuiper-Goodman T, 2004. Risk assessment and risk management of mycotoxins in food. In: Magan N, Olsen M (eds), Mycotoxins in Food Detection and Control. Woodhead Publishing Ltd, Cambridge, pp. 3-27.
Laich F, Fierro F, Martin JF, 2002. Production of penicillin by fungi growing on food products: identification of a complete penicillin gene cluster in Penicillium griseofulvum and a truncated cluster in Penicillium verrucosum. Applied and Environmental Microbiology 68: 1211-1219.

Lenain L, Bonturi M, Koen V, 2002. The fallout from terrorism: security and the economy. Observer No 231/232, 9-11.

Locasto DA, Allswede M, Stein TM, 2004. CBRNE - T-2 Mycotoxin. E-medicine June. http://www.emedicine.com/emerg/topic890. htm (accessed 2/6/06).

Mays LW, 2004. Water Supplies Systems Security. AWWA Products, Denver.

Miller AJ, Hileman CL, Droby S, Paster N, 2005. Science and technology based countermeasures to foodborne terrorism: introduction. Journal of Food Protection 68: 1253-1255.

Paterson RRM, 2006a. Primers from the isoepoxydon dehydrogenase gene of the patulin biosynthetic pathway to indicate critical control points for patulin contamination of apples. Food Control 17: 741-744.

Paterson RRM. Aflatoxins contamination in chilli samples from Pakistan. Food Control, in press.

Paterson RRM, Kelley J, Gallagher M, 1997. Natural occurrence of aflatoxins and Aspergillus flauus (LINK) in water. Letters in Applied Microbiology 25: 435-436.

Paterson RRM, 2006b. Identification and quantification of mycotoxigenic fungi by PCR. Process Biochemistry 41: 1461-1474.

Paterson RRM, Venâncio A, Lima N, 2004. Solutions to Penicillium taxonomy crucial to mycotoxin research and health. Research in Microbiology 155: 507-513.

Paterson RRM, Lima N, 2005. Fungal contamination of drinking water. In: Lehr J, Keeley J, Lehr J, Kingery III TB (eds), Water Encyclopedia. John Wiley \& Sons, New York, pp. 1-7.

Shannon M, 2004. Management of infectious agents of bioterrorism. Clinical Pediatric Emergency Medicine 5: 63-71.

Sharpira R, 2004. Control of mycotoxins in storage and techniques for their decontamination. In: Magan N, Olsen M (eds), Mycotoxins in Food Detection and Control. Woodhead Publishing, Cambridge, UK, pp. 190-223.

Sheppard DC, Ibrahim AS, Edwards Jr JE, 2004. Human mycosis: the role of molecular biology. In: Tkacz JS, Lange L (eds), Advances in Fungal Biotechnology for Industry, Agriculture and Medicine. Kluwer/Plenum Publishers, New York, pp. 361-384.

Skrobek A, Boss D, Défago G, Butt TM, Maurhofer M, 2005. Evaluation of different biological test systems to assess the toxicity of metabolites from fungal biocontrol agents. Toxicology Letters 161: 43-52.

Stark A-A, 2005. Threat assessment of mycotoxins as weapons: molecular mechanisms of acute toxicity. Journal of Food Protection 68: 1285-1293.

Strasser H, Vey A, Butt TM, 2000. Are there any risks in using entomopathogenic fungi for pest control, with particular reference to the bioactive metabolites of Metarhizium, Tolypocladium and Beauveria species? Biocontrol Science and Technology 10: 717-735.

Tucker JA, 2003. Biosecurity: Limiting Terrorist Access to Deadly Pathogens. Peaceworks No. 52, United States Institute of Peace, Washington DC.

de Vries GE, 2000. Fusarium considered to kill coca plants. Trends in Plant Science 5: 417.

Whitaker TB, Johansson AS, 2005. Sampling uncertainties for the detection of chemical agents in complex food matrices. Journal of Food Protection 68: 1306-1313.

WHO, 2003. Laboratory Biosafety Manual. Second edition (revised). Interim guidelines. WHO, Geneva. 